Instituto Internacional de Investigación y Desarrollo Tecnológico Educativo INDTEC, C.A.

DOI: https://doi.org/10.29394/scientific.issn.2542-2987.2017.2.3.16.289-309

OAI-PMH: http://www.indteca.com/ojs/index.php/Revista Scientific/oai

\title{
Liderazgo Situacional y su Influencia en la Gerencia del Docente de Aula en la Carrera de Administración Industrial
}

Autora: Neida del Carmen Molina Alcedo Universidad Fermín Toro, UFT

neicarmolina@gmail.com

Lara, Venezuela

\section{Resumen}

La presente investigación tuvo como objetivo analizar el liderazgo situacional y su influencia en la gerencia del docente de aula, en la carrera Administración Industrial. El estudio de naturaleza descriptiva, de campo, contó con una población de 35 docentes, a quienes se les aplicó un instrumento tipo cuestionario de 21 ítems, modelo escala de Lickert, con tres opciones de respuesta: Siempre, Algunas Veces y Nunca, el cual fue validado y cuya confiabilidad arrojó un resultado de 0.94 medido mediante la fórmula del Coeficiente Alfa de Cronbach. La interpretación de los resultados, permite señalar que los docentes no presentan un estilo de liderazgo situacional en particular y en relación a los procesos administrativos utilizados en su práctica docente, se evidenció que ocasionalmente realizan actividades de planificación, organización, dirección, control y evaluación. Por tanto, se sugiere la capacitación de los docentes en los estilos del liderazgo situacional y los principios gerenciales que coadyuven a mejorar la calidad educativa.

\section{Palabras clave: liderazgo situacional; gerencia; educación universitaria.}




\title{
Situational Leadership and Influence in the Management of Classroom Teacher in the Industrial Management
}

\begin{abstract}
This research aimed to analyze situational leadership and influence in the management of classroom teacher in Industrial Administration career. The study of descriptive nature, field, had a population of 35 teachers, who were applied a questionnaire of 21 items, model scale Likert, with three choices of response type instrument: Never Always, sometimes, and which he was validated and whose reliability yielded a result of 0.94 measured by the formula Cronbach Alfa coefficient. The interpretation of the results, to point out that teachers do not have a style of situational leadership in particular and in relation to administrative processes used in their teaching, it was shown that occasionally perform planning, organization, management, control and evaluation. Therefore, training of teachers in the styles of situational leadership and management principles that help to improve educational quality is suggested.
\end{abstract}

Keywords: situational leadership; management; higher education.

Date Received: 14-09-2016

Date Acceptance: 18-10-2016 


\section{Introducción}

El liderazgo del docente y la gerencia en el aula son aspectos que deben considerarse en cualquier modelo educativo. En el plano universitario la práctica gerencial, así como la utilización de un estilo particular de liderazgo en cada situación del hecho educativo son aspectos que deben valorarse en su justa dimensión; ya que las dificultades que recurrentemente presentan los estudiantes universitarios en su aprendizaje constituyen un motivo constante de preocupación para quienes se dedican a la enseñanza y están comprometidos con el mejoramiento de la calidad de la educación.

Las universidades para responder a las exigencias de la sociedad actual, necesitan estar en sintonía con la actualización permanente. En este sentido requieren de docentes dispuestos al cambio; capacitados en liderazgo y gerencia educativa. Sobre esta premisa se genera el objetivo de la presente investigación: analizar el liderazgo situacional del docente y su influencia en la gerencia del aula, en la carrera Administración Industrial del Instituto Universitario de la frontera, San Cristóbal, estado Táchira.

La capacitación docente en liderazgo situacional y gerencia, implica un compromiso individual y colectivo por parte de los sujetos que forman parte de cualquier organización educativa. Para Hersey, Blanchard y Johnson (1998), el liderazgo es "el proceso de influir en las actividades de un individuo o grupo en los esfuerzos por alcanzar una meta en cierta situación" (pág. 99). Esta distinción permite que una persona tenga influencia sobre otra, tal como es la relación que se presenta entre profesores y estudiantes. El planteamiento expuesto anteriormente conlleva a formular la siguiente interrogante: ¿Cuáles son las características del liderazgo situacional y su relación con la gerencia del docente de aula en la carrera Administración Industrial? 


\section{Teoría y Conceptos}

\subsection{Liderazgo}

Las organizaciones exitosas son aquellas que entienden y sostienen mediante la acción, que lo importante para ellas es el desarrollo y crecimiento de su personal y a sus dirigentes como líderes, para lograr cambios organizacionales en positivo, liberando el potencial total de sus seguidores. En tal sentido, es necesario conocer la dimensión teórica de liderazgo que proponen algunos especialistas en el tema, los cuales se nombran a continuación:

Se inicia con Hunter (2000), quien define el liderazgo como "el arte de influir sobre la gente para que trabaje con entusiasmo en la consecución de objetivos en pro del bien común" (pág. 12). De acuerdo con este autor, en el caso del presente estudio una habilidad que deben poseer los docentes para influir en los estudiantes para lograr los objetivos de la asignatura y por ende los de la institución universitaria en beneficio de todos. Por su parte, Lusier y Achúa (2002), definen el liderazgo como "un proceso en el cual influyen líderes sobre seguidores y viceversa, para lograr los objetivos de una organización través del cambio" (pág. 6); de esta manera se percibe la impostergabilidad de la influencia que se produce recíprocamente entre líderes y seguidores, proceso resultante de la persuasión que ejerce un buen líder para gestionas sus acciones.

Otra de las definiciones de liderazgo que son claves en el estudio es la de Hersey y Blanchard (2006), quien define el liderazgo: "el proceso dinámico de influir sobre los demás para seguir tras el logro de un objetivo común. El comportamiento de un individuo también puede influir cuando éste, está involucrado en la dirección de las actividades de los seguidores" (pág. 55). De estas definiciones se desprende algo muy importante, el líder debe en todo momento tener presente, que su actuación no va en función de su persona sino de un individuo con capacidad de actuar en un momento dado. 


\subsection{Liderazgo Situacional}

El estilo de liderazgo más eficaz es aquel que se adapta a los colaboradores en cada situación; en otras palabras, ejercer un liderazgo adecuado a las necesidades del equipo. Así, el liderazgo situacional busca mantener un equilibrio entre los tipos de comportamiento que ejerce un líder, para adaptarse al nivel de desarrollo de su equipo de trabajo. Al respecto, Hunter (ob.cit), señala que el líder situacional "es la persona que maneja la máxima calidad de lo que se quiere lograr a través de tareas estructuradas que favorecen al líder en toda situación" (pág. 141). De lo antes expuesto se infiere que, la relevancia está en el grado de estructuración presente en el trabajo a ejecutar, lo cual influirá proporcionalmente en los resultados.

Por otra parte, mientras más habilidad tenga el líder para comunicarse con su personal, mayor será la influencia que tenga; es decir, la posición formal de poder que ocupa el docente, para que sea efectiva requiere de una alta estructuración del trabajo a ejecutar y de una relación docente - estudiantes altamente positiva. De allí, el liderazgo situacional es una teoría de la contingencia que se enfoca en los seguidores, al respecto, Hersey y Blanchard (2006), señalan: "las habilidades especificas a desarrollar en el líder implican actitudes y aptitudes. El líder adecua su actitud para comunicarse y manejar su aptitud para manejar contingencias" (pág. 125). En esencia este modelo establece la necesidad de un estilo a ser utilizado por el líder, a las diferentes situaciones que debe enfrentar, en particular a las características de la persona que será objeto de la acción de liderazgo.

En este contexto, las organizaciones dependen, para crecer y perdurar del liderazgo de sus dirigentes, para lo cual se amerita reunir cuatro condiciones; compromiso con la misión, comunicación de la visión, confianza en sí mismo e integridad personal. Básicamente el liderazgo consiste en una forma de ser, pues el líder se va formando día a día en la pasión por la misión, en la acción. Además, no solamente debe delegar responsabilidades, sino que 
debe expandir el poder hacia otros, ser humilde, considerarse sustituible, así realizar acciones eficientes en forma conjunta y sin desconexiones. Cabe destacar, que trasladar el liderazgo situacional al campo de la gerencia de aula, implica capacitar al docente como un líder que dirija, guíe, apoye, oriente y delegue adecuadamente el trabajo con sus estudiantes.

\subsection{Estilos del liderazgo situacional}

El modelo de liderazgo situacional de Hersey y Blanchard (2006): propone que el responsable de dirigir un grupo u organización varíe su forma de interactuar y abordar las tareas en función de las condiciones de sus colaboradores. Es un método útil para aquellos directores de equipos que no encuentran respuestas en los modelos que toman como ejemplo a grandes líderes de la historia. Frente a eso, en el modelo Hersey-Blanchard se encuentra un modo simple de diagnosticar a su grupo y comenzar a mejorar su rendimiento. El mismo establecía que el rendimiento de los grupos de trabajo depende de una combinación acertada entre el modo de interactuar del líder con sus subordinados y las condiciones en las que se puede ejercer influencia y control en que cada situación concreta, desarrollando una diferenciación en sus estilos.

Estilo Dirigir, el líder explica claramente las reglas, establece objetivos, controla con firmeza cuando es necesario. Proporciona dirección y supervisión a los subordinados con poca motivación y debilidades en su capacitación. Estilo Guiar, el líder explica como la tarea se relaciona con los objetivos importantes de la organización, anima a sus seguidores, es un orientador, proporciona formación y entrenamiento cuando se necesita, se toma tiempo para desarrollar las habilidades de su personal con paciencia. Estilo Apoyar, el líder contribuye a fortalecer la autoestima de su equipo. Incentiva el trabajo en equipo del personal capacitado que esta desmotivado. Estilo Delegar, el líder permite que otros asuman la responsabilidad que consideren pueden 
manejar. Delega apropiadamente en las personas capacitadas y altamente motivadas.

Los autores Hersey y Blanchard, explican que la aplicación de un estilo determinado depende de la capacidad de cada individuo. Definen la capacidad como la suma de motivación personal más habilidades en la actividad asignada. Las habilidades guardan relación con el grado de experiencia, el nivel de entendimiento, educación y conocimiento específico de la tarea encomendada; Asimismo, señalan que, para un liderazgo situacional efectivo, se debe dirigir a las personas con poca habilidad y poca motivación; en caso de tener mucha motivación y poca habilidad es necesario guiarlas; si su situación es de mucha habilidad y poca motivación se valora su talento apoyándolas para que se integren al trabajo en equipo.

\subsection{Características del gerente de aula como líder situacional}

\subsubsection{Habilidades humanísticas}

Los líderes requieren habilidades humanísticas que les permitan en un momento dado prever el futuro; inspirar a los miembros de la organización y trazar la ruta y/o camino que ésta seguirá. El líder también debe ser modelo de valores, porque sin estos no se pueden pensar en la calidad de recursos humanos, reforzar su ética, es un valor impostergable de su desempeño. Asimismo, en el ámbito universitario los líderes deben preocuparse por capacitar y actualizar a sus liderados, así lograr un personal docente calificado, honesto y proactivo con dominio de nuevas tecnologías, que coadyuven a modernizar la institución y por ende maximizar el producto educativo, en tal sentido es necesario precisar en forma continua las dimensiones del liderazgo.

Al respecto, Dilts (2003): coincide al reseñar las dimensiones del liderazgo, destacando: 1. La capacidad del líder para hacer uso eficaz y responsable del poder con sentido humanístico: Capacidad para comprender que los seres humanos tienen diferentes motivaciones en diferentes 
momentos y situaciones, esto conlleva a un profundo conocimiento de los individuos sobre las motivaciones de sus seguidores, siendo capaz de aplicar estos saberes en diferentes situaciones. 2. Capacidad para actuar a favor del desarrollo de un clima armónico, direccionado a dar respuestas a las motivaciones del personal y gerencia. Esto tiene que ver con el líder y el ambiente y/o clima que el promueva; también implica la intensidad de la motivación.

\subsubsection{Motivación de logro}

El gerente de aula en el desempeño de su ejercicio profesional debe incentivar la motivación de los estudiantes, saber identificarse con el tipo de motivación que se requiere de acuerdo al comportamiento organizacional le proporciona a la gerencia una buena integración de su equipo de trabajo y que se manifieste un clima organizacional idóneo.

Desde esta perspectiva, el fundamento conceptual de la motivación de logro se basa en una necesidad planteada por Farlland y otros (1996), en su teoría de las necesidades sociales, la necesidad de logro. En su contenido esta orientación motivacional expresa "cómo las personas realizan las actividades por el deseo obtener éxito en las acciones y persistir hasta lograrlo, experimentando con ello una sensación de orgullo" (pág. 74). En otras palabras, la motivación de logro representa la orientación motivacional que asumen las personas hacia la consecución del éxito en lo que realizan, la misma está determinada por el comportamiento de estas dos tendencias y resulta decisiva en la elección de la ruta psicológica que se sigue para lograr los objetivos propuestos.

\subsubsection{Comunicación en el aula}

La acción comunicativa representa un papel de suma importancia para todo docente, cabe destacar que, aunque cumpla otras funciones dentro de 
alguna institución educativa, no debe obviar que es esencialmente un ser humano que participa directamente en el desarrollo humano de las nuevas generaciones, su misión es importante porque gracias a su función es posible la evolución de la especie humana. Según la perspectiva constructivista, la comunicación educativa constituye el proceso mediante el cual se estructura la personalidad del educando, lográndose a través de las informaciones que este recibe y reelaborándolas en interacción con el medioambiente y con los propios conceptos construidos.

\subsubsection{Trabajo y aprendizaje en equipo}

Las nuevas tendencias laborales conducen a pensar en los equipos como una forma habitual de trabajo, en este sentido Espinosa (2007): "el trabajo en equipo se refiere a la serie de estrategias, procedimientos, y metodologías que utiliza un grupo humano para lograr las metas propuestas". (pág. 1). Como consecuencia el trabajo en equipo origina una integración armónica de los miembros del equipo de trabajo, se compartirán las responsabilidades que se realicen en forma coordinada y apuntarán a un objetivo común en pro del éxito.

En cuanto a las características del trabajo en equipo el autor señala: que permite la armonía y la integración en la ejecución de las actividades, apela a la responsabilidad por parte de los miembros del equipo, en cuanto a las acciones deben estar planificadas y bien coordinadas. El trabajo en equipo permite un desempeño armónico de las acciones que contribuirá de manera efectiva el desempeño. Por ello, el aprendizaje en equipo es importante y necesario dentro de cualquier organización, consiste en adquirir nuevos conocimientos mediante el trabajo en conjunto o cooperativo donde los integrantes aprenden por medio del compartir y la unidad. 


\section{Gerencia}

De manera general se denomina gerencia a la dirección de una organización institucional. La gerencia delega a su vez dirección en diferentes áreas y departamentos. La gerencia tiene la función de orientar los esfuerzos y recursos en una determinada dirección para el logro de objetivos. En relación a la definición del término gerencia, Sisk y Sverdlik (2002), señala:

El término gerencia es difícil de definir: significa cosas diferentes para personas diferentes. Algunos lo identifican con funciones realizadas por empresarios, gerentes o supervisores, otros lo refieren a un grupo particular de personas. Para los trabajadores; gerencia es sinónimo del ejercicio de autoridad sobre sus vidas de trabajo... (pág. 32).

Con base a lo expresado por este autor, la gerencia tiene que ver con las funciones que ejecuta una persona dotada de autoridad. El gerente en su labor diaria toma decisiones que impactan el grupo de personas que dirige, por ello es de suma importancia que mantenga una buena comunicación con sus colaboradores. Lograr una buena comunicación suele ser una de las más importantes tareas de la gerencia, y es por ello que el gerente debe tener reuniones individuales con cada integrante del grupo, así como también con todo el equipo o grupo.

\subsection{Funciones de la gerencia}

La gerencia es un proceso dinámico que se nutre de las siguientes habilidades técnicas: (a) Planificación, en la que se diagnostican las debilidades, fortalezas, oportunidades y amenazas de la organización, para reflexionar y definir el plan de acción que contiene los objetivos generales y específicos que se desean lograr, los valores, la visión y la misión institucional. La planificación debe ser flexible y sujeta a modificaciones en el curso de su ejecución; (b) Organización, para poder llevar a la práctica y ejecutar los planes, una vez que estos han sido preparados, es necesario crear una 
organización. Es función de la gerencia determinar el tipo de organización requerido para llevar adelante la realización de los planes que se hayan elaborado. La clase de organización que se haya establecido, determina, en buena medida, el que los planes sean apropiada e integralmente apropiados. De igual manera, la (c) Dirección, esta función gerencial va de la mano con habilidades de liderazgo, coordinación y ejecución, a pesar de que cada uno de estos términos tiene una connotación diferente, todos ellos indican claramente que esta función gerencial tiene que ver con los factores humanos de una organización. Es como resultado de los esfuerzos de cada miembro de una organización que ésta logra cumplir sus propósitos de ahí que dirigir la organización de manera que se alcancen sus objetivos en la forma más óptima posible, es una función fundamental del proceso gerencial.

Por último, y no la menos importante, (d) el Control, fase del proceso gerencial que tiene el propósito, inmediato de medir, cualitativa y cuantitativamente la ejecución en relación con los patrones de actuación; además determina la necesidad de tomar acciones correctivas o remediales que encauce la ejecución cónsona con las normas establecidas. La función de control es ejercida continuamente, y, aunque está relacionada con las funciones de organización y dirección, está más íntimamente asociada con la función de planificación. La acción correctiva del control da lugar, casi invariablemente, a un replanteamiento de los planes; es por ello que muchos estudiosos del proceso gerencial consideran ambas funciones como parte de un ciclo continuo de planeamiento-control-planeamiento.

\subsection{Gerencia educativa}

La actividad docente forma parte de la gerencia educativa y está sustentada en principios generados de los postulados modernos del desarrollo organizacional, los procesos administrativos, las estrategias y las tácticas gerenciales. De allí, que ineludiblemente para lograr una gerencia de aula 
efectiva, los docentes en su rol de gerentes deben considerar los elementos antes mencionados. Por ello a continuación, se describen aspectos relevantes relacionados a la gerencia, que a discreción de la autora de este estudio pueden ser aplicados a la gerencia de aula.

En este sentido, la gerencia para Fermín (2007): viene del latín "genere" que significa dirigir y se identifica con empresas que realizan actividades de planificación, organización, ejecución y control para el uso eficaz de los recursos humanos, físicos, financieros y tecnológicos, con el propósito de lograr objetivos económicos y generar beneficios sociales.

De acuerdo a lo anteriormente planteado, se deduce que la gerencia está destinada a utilizar los recursos de las instituciones de manera eficiente y eficaz, creando beneficios colectivos a la comunidad donde esté ubicada la organización. De allí, que Manes (2003): expresa que la gerencia educativa es un proceso de administración de una institución escolar a través del cumplimiento de un conjunto de habilidades gerenciales orientadas a planificar, organizar, coordinar y evaluar la gestión estratégica de las actividades necesarias para alcanzar la eficiencia pedagógica, administrativa, comunitaria de la institución educativa.

\section{Caracterización del profesional de la docencia}

Referir la importancia que debe tener la formación de un docente universitario para desempeñar las funciones básicas, debe prepararse para atender los requerimientos de la docencia, investigación y extensión; estos tres elementos funcionan en interrelación, puesto que la docencia proyecta situaciones de investigación dirigidas a generar cambios dentro del contexto educativo institucional o en el entorno donde funciona.

La ausencia de actualización docente en las instituciones universitarias, se traduce en una limitante para el éxito de la práctica educativa en el aula. En el marco de la actualización docente Birgin y otros (1998), señalan: "...la 
andragogía es un enfoque educativo a considerar en la formación del docente universitario, es un elemento absolutamente importante para la transformación exitosa del clima educativo que debe prevalecer en la universalidad del pensamiento" (pág. 147); lo expresado por los precitados autores, demuestra que la preparación del docente universitario requiere de elementos pedagógicos, pero también de la andragogía como estrategia para orientar el aprendizaje del estudiante universitario.

Como producto de esta formación, se va a proyectar en el estudiante universitario, a través, de su capacidad y perspectiva de integración al contexto social constituido por el recurso humano exigido en el país, con la intencionalidad adherida de conjugar una variedad de profesiones orientadas hacia el logro de los objetivos comunes. La educación universitaria está centrada en la relación efectiva entre la formación del docente universitario y la aplicación de sus conocimientos, habilidades y destrezas en la formación del estudiante.

\subsection{Formación profesional}

Al hablar de profesores, el enfoque automático se orienta hacia los sistemas tradicionales de educación; sin embargo, las exigencias contemporáneas hacen que todo educador este en constante mejora continua, porque para ser un docente, el rol no está solamente en enseñar, sino enseñar a aprender. Díaz y otros (2001), señalan que: “...la formación docente no puede enfocarse en un plano individual, porque no permite superar la imagen espontánea y simplista de la enseñanza" (pág. 3). Acción que obliga al docente a integrar en su quehacer cotidiano de aula un marco teórico conceptual sobre la aplicación de relaciones, definir su propia presencia como docente, analizando sus deficiencias y como minimizarlas, al igual, las opciones de obtener mejores procedimientos. 


\subsection{La Formación del docente universitario}

En estos momentos, la formación docente conlleva a plantear algunos interrogantes y reflexiones; ya que la misma asume un rol protagónico como forma de solucionar gran parte de los males por los que atraviesa la educación, entre las que se destaca la acción gerencial, más aún en que se propone una nueva forma de considerar la administración educativa universitaria. Por otra parte, el ingreso de medios tecnológicos al ámbito escolar, resultaría inútil si únicamente se los usara en forma lúdica para convencer a sus alumnos de que aprender es tan divertido como mirar televisión, esta incorporación de los avances científicos conducen a la necesidad de adecuar las estructuras administrativas y académicas a esta realidad y por ende, los procesos administrativos inherentes a la gerencia deben responder a tales exigencias. Machado y otros (1999), plantean:

En la educación actual el individuo postmoderno, desenvuelto de necesidades pasajeras y aleatorias, ha olvidado que la libertad era no otra cosa que la potestad de cambiar de cadenas y la propia cultura, algo más que una pulsión satisfecha. Lo cual induce a que los procesos educativos tienen que ser cambiantes y por lo tanto la calidad del docente debe girar en función a tales cambios sociales (pág. 231).

Del planteamiento anterior se desprende, la reivindicación gremial que exija el derecho a la capacitación permanente del docente, pero a la vez es un deber del docente asumir su compromiso individual de formación, porque el educador requiere herramientas metodológicas, didácticas, para garantizar que los estudiantes universitarios aprendan más y mejor, se cuestionan los modos de implementarlas y la falta de medios para redimensionarlas y hacerla más adecuada con esto se pretende enfatizar la necesidad de un seguimientos de la capacitación docente, es decir, en su relación directa con el mejoramiento de la propuesta de enseñanza y su implantación en el aprendizaje de los estudiantes. 


\section{Contexto educativo}

Con base a las vivencias del ejercicio docente, se puede señalar que el desarrollo profesional de las instituciones educativas no es una tarea común; y su concepción e instrumentación no puede hacerse sin ideas, sin talento y sin experiencia; no puede dejarse a la improvisación; es una función para especialistas que requieren cubrir criterios de idoneidad soluciones a los problemas de la institución. Sin embargo, la problemática de la capacitación y formación docente en educación superior, se puede plantear desde múltiples aproximaciones disciplinarias, ya que se requiere la participación de diversos profesionales para la atención de las áreas del conocimiento.

Se puede detectar, que en la inmensa mayoría de las instituciones de educación superior existe una formación universitaria rígida, con currículo poco flexibles y ausencia innovadora en los procesos educativos, que impone un perfil predominante del profesor de corte tradicional, que privilegia el aprendizaje memorístico y la reproducción de saberes, con base en una praxis acrítica, fundamentalmente entendida como un conjunto de principios que orienta irreflexivamente a los profesores en la traducción de objetivos educativos a prácticas pedagógicas concretas.

En este contexto, en opinión de la investigadora, es necesario resaltar que para iniciarse en la docencia a nivel superior, además de cubrir los requisitos de ingreso propio en cada institución educativa se debe contemplar que una característica primordial deber ser una actitud cognitiva de innovación y de creatividad, es decir, que tenga capacidad de aprender a aprender y de cierto grado de sociabilidad, ya que el desempeño laboral requiere desarrollar la relación con personas y su posición con el entorno.

Queda claro que para toda institución de educación superior el eje central para el mejoramiento de la calidad educativa es la superación y mejoramiento del personal académico, lograda a través de la implementación de estrategias de actualización y formación docente, que varían dependiendo 
de las políticas institucionales, en las que se debe promover que los docentes desarrollen más su capacidad en el ámbito profesional y brindarles alternativas de innovación docente con el fin de consolidar cuerpos académicos.

\section{Metodología}

En función de los propósitos que identifican el presente estudio, se enmarcó dentro de la concepción paradigmática cuantitativa porque aborda procedimientos que buscan describir la realidad que caracteriza el liderazgo situacional y la gerencia de aula, lo cual a juicio de Cerda (1991): este paradigma "tiende a detallar procedimientos para abordar la realidad al interconectar aspectos que dan sentido globalizador del hecho estudiado" (pág. 12). Desde esta concepción, se asumió el tipo de investigación descriptiva con un diseño de campo, por cuanto sus concepciones concuerdan con las pretensiones del estudio.

De este modo, responde al tipo de investigación de campo que según Arias (2003): busca la "recolección de datos directamente de la realidad donde ocurren los hechos sin manipular o controlar variable alguna..." (pág. 48). En atención a la visión descrita, se consideró este tipo de investigación en virtud de indagar directamente la realidad, confrontada por los docentes que laboran en la carrera de Administración Industrial del Instituto Universitario de la Frontera. San Cristóbal, estado Táchira, a fin de recopilar la información de los hechos tal como se presentan, sin ningún tipo de manipulación, la cual permitirá conocer a fondo el problema.

Igualmente, la investigación es de carácter descriptivo, porque a juicio de Hernández y otros (2006): "consiste en indicar lo que es y se interesa por las condiciones o relaciones existentes; las prácticas que predominan, creencias y actitudes, los procesos que suceden o las tendencias que están desarrollándose" (pág. 11). En tal sentido, se apoyó en la descripción sistemática de la situación, con el propósito de detallar los hechos tal como 
suceden, es decir, se plantean aquellas acciones relacionadas con el liderazgo situacional y su influencia en la gerencia de aula. Estos estudios, buscan explicar las propiedades, las características y los perfiles de personas, grupos, procesos, otros, por medio de mediciones, recolección evaluaciones de datos sobre diversos conceptos (variables), aspectos o dimensiones del fenómeno a investigar, de esta manera, se describe lo que se investiga.

En este orden de ideas, Hernández y otros (2006), al referir sobre los diseños no experimentales: "...lo que hacemos en la investigación no experimental es observar fenómenos tal cual se dan en su contexto natural para después analizarlos" (pág. 205). En consecuencia, el diseño de la investigación corresponde al plan que se desarrolló para obtener la información que se requería en la investigación. En este caso de estudio, se tomaron los hechos de la realidad, y se determinaron las situaciones que tenían que ver con las variables y sus respectivas dimensiones, así como los factores que en ella incidían, medidos directamente a través de indicadores, por tal razón su diseño es no experimental, debido a que no hubo manipulación de las variables.

\section{Conclusiones}

La conclusión principal que se puede extraer de este estudio, es que los docentes involucrados en la investigación no revelan un liderazgo situacional en habilidades humanísticas, motivación de logro, comunicación en el aula, trabajo y aprendizaje creativo, estilo dirigir, estilo guiar, estilo, apoyar y estilo delegar, salvo algunas excepciones; sin embargo; la mayoría de los docentes expresan que siempre emplean la comunicación en el aula para asegurar el éxito en el trabajo escolar. Igualmente se observa que un porcentaje significativo de docentes se identifican con los estilos de dirigir y guiar a los estudiantes. 
En líneas generales los docentes del Instituto Universitario de la frontera de la carrera Administración Industrial no asumen un liderazgo situacional particular, debido probablemente a las diferentes variables que inciden en su conducta. En relación a la gerencia de aula, no se refleja en los docentes una práctica constante relativa a la planificación, organización, dirección, control y evaluación. En lo que respecta a la planificación se evidencia por una parte que los docentes le dan importancia al logro de objetivos de las asignaturas, pero descuidan el logro de las metas institucionales.

En la organización de la metodología y normas para el logro de objetivos y trabajo grupal existen debilidades notables. En cuanto a la dirección, un porcentaje importante de los docentes, orienta la práctica educativa bajo criterios pedagógicos de la enseñanza activa, sin embargo, dejan de lado la dirección de actividades individuales para asegurar cambios significativos en la capacitación de los estudiantes. Situación similar se presenta en los procesos administrativos de control y la evaluación.

Existe una incidencia significativa entre los estilos del liderazgo situacional: dirigir, guiar, apoyar, delegar y los principios administrativos de la gerencia de aula: planificación, organización, dirección, control y evaluación. El fortalecimiento de la gerencia de aula depende principalmente del docente, de sus cualidades humanas y de su rol de líder. Queda clara la percepción del docente, sobre la necesidad de adquirir un liderazgo situacional, en su rol de gerente de aula; como facilitador pedagógico en el hecho educativo, incluyendo estrategias didácticas que permitan al estudiante de Administración Industrial alcanzar niveles de conocimiento significativo y profundo, sin caer en lo memorístico y superficial.

\section{Referencias}

Arias (2003). Guía para la elaboración de proyectos de investigación.

Caracas. 
Birgin, y otros (1998). Las instituciones de formación docente frente a la reforma: estrategias y configuraciones de la identidad, en Revista Propuesta educativa. Año 9, № 5 . FLACSO Novedades Educativas. Buenos aires.

Cerda (1991). Elementos de la investigación. 1a. edición. Editorial El Búho LTDA. Santa Fe de Bogotá, D. C.

Dilts, K. (2003). Perspectivas del liderazgo. México: Editorial Morata.

Díaz, E., y otros (2001). Estrategias Docentes para un Aprendizaje Significativo. Bogotá: McGraw-Hill.

Espinosa (2007). Gestión de instituciones escolares: las variables claves de las relaciones interpersonales y su influencia sobre el rendimiento escolar. Tesis para optar al grado de Magíster en Ciencias de la Ingeniería, Pontificia Universidad Católica de Chile.

Farlland, L., Childress, J., y Senn, L. (1996). Liderazgo para el siglo XXI. Editorial McGraw-Hill. Colombia.

Fermín, J. (2007). Herramientas de participación ciudadana. Caracas: Fundación Escuela de Gerencia Social.

Hernández, y otros (2006). Metodología de la investigación. México: Edit. McGraw-Hill.

Hersey, M., y Blanchard, P. (2006). Teoría del liderazgo situacional. Madrid: Ediciones Pirámides.

Hersey, M. Blanchard, y Johnson (1998). Teoría del liderazgo. Madrid: Ediciones Pirámides.

Hunter, J. (2000). La Paradoja: Un relato sobre la verdadera esencia del liderazgo. Empresa Activa. Barcelona.

Lusier, y Achúa (2002). Liderazgo. México DF: Thomson. (B. Pública de Lima) 303.34 L99. 
Manes (2003). Gestión estratégica para instituciones educativas. Guía para planificar estrategias de gerenciamiento institucional. Buenos Aires: Edic. Granica.

Machado, y Otros (1999). Preparación del docente universitario. Editorial IUPEMAR. Maracay, estado Aragua, Venezuela.

Sisk, y Sverdlik (2002). Administración y gerencia de empresas. Cincinnati, OHIO, USA: South Westerm Publishing Co (10ma reimpresión). 


\section{Neida del Carmen Molina Alcedo}

e-mail: neicarmolina@gmail.com

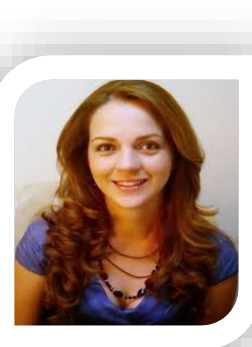

Nacida en pregonero estado Táchira, San Cristóbal, Venezuela. Estudia el doctorado en Educación en la universidad Pedagógica Experimental Libertador. San Cristóbal (2016). Magister Scientiarum en Educación Superior mención Docencia Universitaria. Universidad Fermín Toro (2014). Especialista en Gerencia Educativa, Universidad Santa María (2009). Licenciada en Educación Integral, Universidad Nacional Abierta (2007) Técnico Superior Universitario en Administración Industrial, Instituto Universitario de la Frontera. (2002). Estudios de Primaria en el Colegio Santa Mariana de Jesús, Media General en el Liceo Liborja y Mora. Actualmente se desempeña como docente de la asignatura Metodología de la Investigación de la Universidad Nacional Experimental del Táchira.

El contenido de este manuscrito se difunde bajo una Licencia de Creative Commons ReconocimientoNoComercial-Compartirlgual 4.0 Internacional 